\title{
Blackbody emission under laser excitation of silicon nanopowder produced by plasma-enhanced chemical-vapor deposition
}

\author{
J. Costa and P. Roura \\ Department of Enginyeria Industrial, GRM, Universitat de Girona, E17071 Girona, Catalonia, Spain
}

J. R. Morante and E. Bertran

Department Física Aplicada i Electrònica, Facultat de Física, Universitat de Barcelona, Av. Diagonal 647, E08028 Barcelona, Catalonia, Spain

(Received 3 March 1998; accepted for publication 10 March 1998)

\begin{abstract}
Previous results concerning radiative emission under laser irradiation of silicon nanopowder are reinterpreted in terms of thermal emission. A model is developed that considers the particles in the powder as independent, so under vacuum the only dissipation mechanism is thermal radiation. The supralinear dependence observed between the intensity of the emitted radiation and laser power is predicted by the model, as is the exponential quenching when the gas pressure around the sample increases. The analysis allows us to determine the sample temperature. The local heating of the sample has been assessed independently by the position of the transverse optical Raman mode. Finally, it is suggested that the photoluminescence observed in porous silicon and similar materials could, in some cases, be blackbody radiation. (C) 1998 American Institute of Physics.
\end{abstract}

[S0021-8979(98)00112-1]

\section{INTRODUCTION}

The generation of powders during plasma processing operations has attracted much attention because of its negative effect on the production yield in the microelectronics industry. ${ }^{1,2}$ The study of the structure of the powder particles (nanometric size and high chemical purity) has allowed them to be considered as good raw material for nanostructured ceramics (membranes and composites). So, at present, the production of nanoparticles of a number of compounds ( $\mathrm{Si}$, $\mathrm{SiC}, \mathrm{Si}_{3} \mathrm{~N}_{4}, \mathrm{BN}$ ) using plasma technology is an active field.

During the last 4 years, the properties and structure of silicon nanoparticles grown by plasma-enhanced chemical vapor deposition (PECVD) have been reported by several authors. ${ }^{3}$ The most salient property is their unusual radiative emission when excited by a laser beam. ${ }^{4,5} \mathrm{We}$ have found two independent radiative processes, $A$ and $B$, depending on the pressure of the surrounding gas. When excited under vacuum, emission $A$ is dominant. It has a high supralinear dependence on the laser intensity, $I_{L}$ of the form

$$
I_{\mathrm{PL}} \propto I_{L}^{r},
$$

with $r$ as high as 9. Additionally, this emission is quenched when the gas pressure increases. This quenching follows an exponential dependence:

$$
I_{\mathrm{PL}}=I_{0} e^{-P / P_{0}},
$$

with $P_{0}$ of the order of several pascals. This means that emission $A$ is unmeasurable at atmospheric pressure, where only emission $B$ remains. This second radiative emission is pressure independent and increases linearly with the laser power.

The occurrence of radiative emission in Si near the visible region of the spectrum together with the extraordinary characteristics of emission $A$ have led us to study it in great detail. We have attempted to explain it in terms of photoluminescence, because we believed that it had a similar origin to that of the luminescence of porous silicon. ${ }^{6,7}$ In this case, the luminescence near the visible is attributable to the nanometric structure of the material. We proposed a phenomenological model to explain the supralinear dependence of Eq. (1). ${ }^{8}$ The emitting levels were reached through a multistepmultiphonon excitation process. This model explained as well the complicated dynamics of the emission. Within this framework, the pressure dependence of Eq. (2) was simply the result of a similar pressure dependence on the dynamic parameters of each level. ${ }^{9}$ Furthermore, in our latest paper ${ }^{10}$ we demonstrated that the effect of the gas was linked to the energy its molecules were able to extract from the powder after every collision with the particles. Despite the successes of this model, the physical origin of the luminescence remained obscure.

Here we propose an alternative explanation for emission $A$. We analyze it by considering that it is simply due to the blackbody emission of the particles. Under vacuum the energy absorbed from the exciting laser can be dissipated only by radiation whereas, at higher pressures, the gas collisions offer a more efficient dissipation path and the radiation emission is quenched. On the other hand, the nonlinear dependence on laser power is easy to understand in view of the Planck and Stephan-Boltzmann laws. These relationships are developed quantitatively and compared with the experimental results (most of which have been published already). New correlations are established that highlight the predictive power of the new explanation. On the basis of the agreement between theory and experiment, we conclude that emission $A$ is due, indeed, to the thermal radiative emission of the particles. 


\section{EXPERIMENT}

The silicon powder was produced in a rf reactor where a square wave modulatated discharge of silane was established. As obtained, the powder consists of spherical particles with diameters ranging from 10 to $200 \mathrm{~nm}$ and is highly hydrogenated. Annealing at moderate temperatures ( $T=800 \mathrm{~K})$ induces hydrogen desorption. The particles remain amorphous and their shapes do not change significantly. Recrystallization only takes place at much higher temperatures (around $1100 \mathrm{~K}) .^{11}$

The radiative emission was excited by the $488 \mathrm{~nm}$ line of an argon laser which was focused on a spot of a few $\mathrm{mm}^{2}$ on the sample surface. The sample was placed inside a closedcircuit He cryostat that allowed control of the temperature and the gas pressure. At the usual excitation conditions the hydrogen evolves from the powder in a few minutes. ${ }^{12}$ This means that the radiative emission comes from dehydrogenated amorphous silicon particles.

The Raman analysis was performed with the $514 \mathrm{~nm}$ line of an Ar laser focused with a microscope system that produced a spot of about $10 \mu \mathrm{m}$ in diameter on the Si powder sample. The sample was mounted in a cylindrical vacuum chamber of $70 \mathrm{~mm}$ in diameter where the minimum pressure reached was $10^{-2} \mathrm{~Pa}$.

\section{THEORY}

Consider an isolated spherical particle suspended in a gas. If we irradiate it with a laser beam, then the particle temperature will change depending on the balance between the energy absorbed and the energy dissipated. If the particle is small enough, its temperature, $T_{p}$, will be homogeneous and follow the equation:

$$
\frac{4}{3} \pi r^{3} \rho c \frac{d T_{t}}{d t}=\pi r^{2} Q_{\mathrm{abs}} I_{L}-4 \pi r^{2}\left(q_{R}+q_{K}\right),
$$

where $r$ is the radius, $\rho$ the density, and $c$ the specific heat; $Q_{\text {abs }}$ is the absorption efficiency and $I_{L}$ the laser intensity. Finally, $q_{R}$ and $q_{K}$ are the heat fluxes due to the radiative emission and thermal conduction through the gas, respectively.

The radiative emission is given by the StephanBoltzmann law:

$$
q_{R}=\epsilon_{i} \sigma\left(T_{p}^{4}-T_{R}^{4}\right),
$$

where $\sigma$ is the Stephan-Boltzmann constant and $\epsilon_{i}$ is the integrated emissivity of the particle; $T_{R}$ is the radiation temperature around the particle.

Concerning the heat dissipation due to the gas, $q_{K}$, we will consider the limiting case where the pressure is so low that the mean free path is greater than the particle size. Then, $q_{K}$ can be calculated approximately by multiplying the number of collisions by the mean energy per molecule in the gas. This procedure gives:

$$
q_{K}=\frac{1}{4 \sqrt{\pi}} \frac{C_{v}}{R} \sqrt{\frac{8 k_{B}}{m}} \frac{P}{\sqrt{T_{G}}} \alpha\left(T_{p}-T_{G}\right),
$$

where $k_{B}$ is the Boltzmann constant, $m$ is the molecular mass, $T_{G}$ is the gas temperature, $c_{v}$ its specific heat at constant volume, and $R$ the universal constant of gases. The parameter $\alpha$ is the "accommodation coefficient" that accounts for the fact that after one collision the gas molecules will not be thermalized to the particle temperature $(\alpha<1)$.

Once the temperature of the particle has been determined, the radiative emission at any wavelength can be calculated according to Plank's distribution:

$$
I_{e}(\lambda)=\epsilon(\lambda) \frac{8 \pi h c}{\lambda^{5}} e^{-h c / \lambda k_{B} T_{p}},
$$

where it is assumed that $\exp \left(h c / \lambda k_{B} T_{p}\right) \gg 1$.

This model has been applied to silicon particles suspended in a silane plasma ${ }^{13}$ where the heating power was supplied by the ions. The high dispersion of the particles ensured, in that case, that they could be considered as independent. However, in our silicon nanopowder the particles are more densely packed, so we should analyze whether the model leading to Eqs. (3)-(5) is still valid. This will be done by comparing the predictions of the model and the experimental results.

\section{THEORETICAL PREDICTIONS AND EXPERIMENTAL RESULTS}

In this section, Eqs. (3)-(6) will be applied to the conditions of the measurements. In order to simplify the analysis, it will be assumed that $T_{R}^{4} \ll T_{p}^{4}$ [Eq. (4)]. Additionally, for the measurements at cryogenic temperatures care has been taken to thermalize the gas with the cryostat walls $\left[T_{G}=T_{R}\right.$ in Eq. (5)]. ${ }^{10}$

\section{A. Spectral shape of the emission}

According to Plank's distribution [Eq. (6)], the spectrum should have a bell-like shape modulated by the emissivity of the material. The peak position would depend on the temperature and hence on the laser intensity. At lower laser intensities, it would be displaced to longer wavelengths and the relative intensity near the visible would diminish.

The experimental spectra measured for two laser intensities are plotted in Fig. 1. The spectra are continuous, as expected. Unfortunately, the spectral range is limited to 2.5 $\mu \mathrm{m}$ and the position of the maxima is not visible. However, the evolution with laser intensity is as expected. Furthermore, the feature we observe around $1 \mu \mathrm{m}$ at the highest intensity is presumably due to the effect of the band gap on the emissivity.

\section{B. Steady-state intensity dependence on laser power}

The formula that gives the steady-state intensity at vacuum conditions is:

$$
I_{e}(\lambda)=\epsilon(\lambda) \frac{8 \pi h c}{\lambda^{5}} \exp \left[-\frac{h c}{\lambda k_{B}}\left(\frac{4 \sigma \epsilon_{i}}{I_{L} Q_{\mathrm{abs}}}\right)^{1 / 4}\right] .
$$

Therefore, for a given wavelength, the logarithm of $I_{e}(\lambda)$ should be proportional to $I_{L}^{-1 / 4}$. Furthermore, the slope should be proportional to $\lambda^{-1}$. 


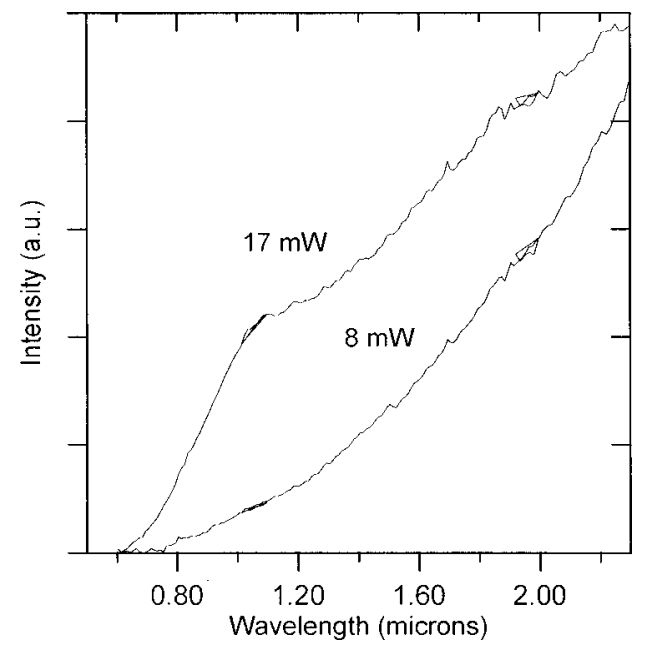

FIG. 1. Emission spectra of the silicon powder at two laser intensities.

In order to test Eq. (7) we have measured the intensity of the emission at two wavelengths $(\lambda=2.1$ and $3.0 \mu \mathrm{m})$ for a range of laser powers. The results are plotted in Fig. 2. The agreement with the expected behavior is excellent. The experimental points are well aligned and the product of the slope and the wavelength is constant. In Sec. IV C we will show that, from these measurements, it is possible to determine the temperature of the particles at each laser power.

\section{Excitation and de-excitation transients}

The dynamics of excitation and de-excitation of the radiative emission in silicon nanopowder has been extensively analyzed elsewhere ${ }^{8}$ within the framework of the multistepmultiphoton excitation mechanism. A number of experiments performed to test the model was done and the agreement was excellent. This explains the confidence of the authors with that interpretation and their reticence to abandon it. Now, several of those results will be used to test the

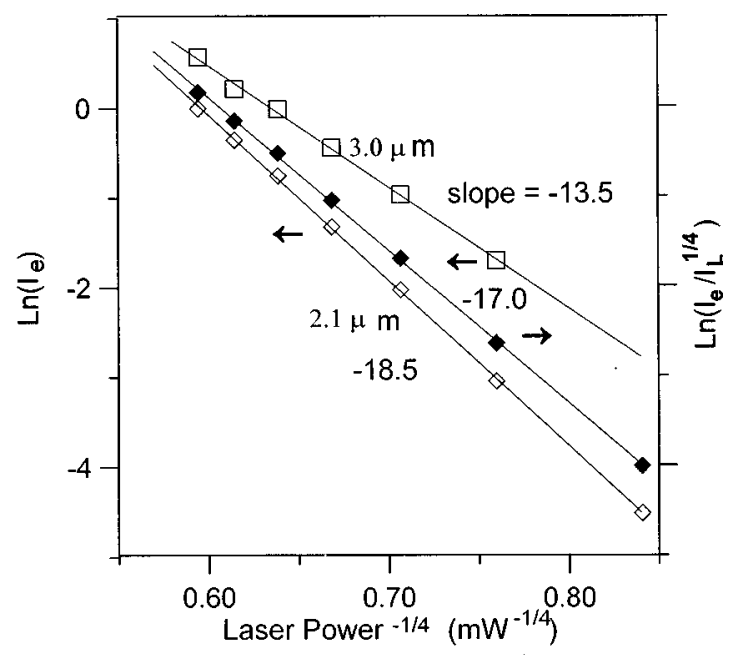

FIG. 2. Dependence of the radiative intensity on the laser power $[(\square) 3.5$ and $(\diamond)(\diamond), 2.1 \mu \mathrm{m}]$. The full symbols correspond to the representation that takes into account the progressive absorption of the laser beam as it penetrates the powder.

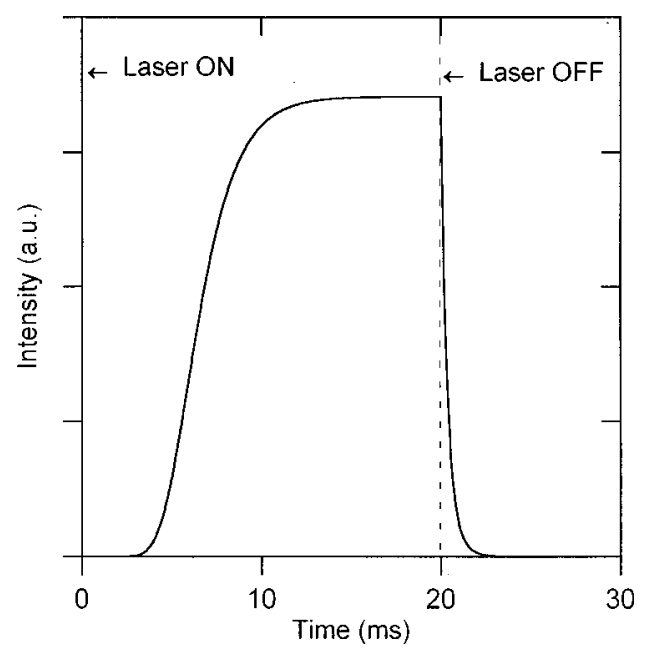

FIG. 3. Excitation and de-excitation transients simulated according to the model of radiative thermal emission of independent particles. The result is comparable to the experimental transients (Ref. 8).

hypothesis of thermal radiative emission, namely: (a) the excitation transient begins with zero slope, (b) the time constant of the excitation is much longer than the decay lifetime (once the laser is turned off), and (c) the decay transient is nonexponential.

A numerical computation of Eqs. (3), (4), and (6) gives the qualitative evolution of the radiative emission over time (Fig. 3). All the characteristic features detailed above are readily reproduced in the calculated dynamics. Our Fig. 3 compares with the experimental result shown in Ref. 8.

We can go further with the model as we can predict the value of the slope for the decay transient just after the laser has been turned off. It is found to be:

$$
\tau=\frac{2}{3} \frac{\lambda k_{B} \rho C}{h c \sqrt{\sigma \epsilon_{i} Q_{\mathrm{abs}}}} I_{L}^{-1 / 2} .
$$

So, at a given wavelength, the decay time constant measured at $t=0$ must be proportional to $I_{L}^{-1 / 2}$. This is just what we find if we analyze the experimental transients (Fig. 4).

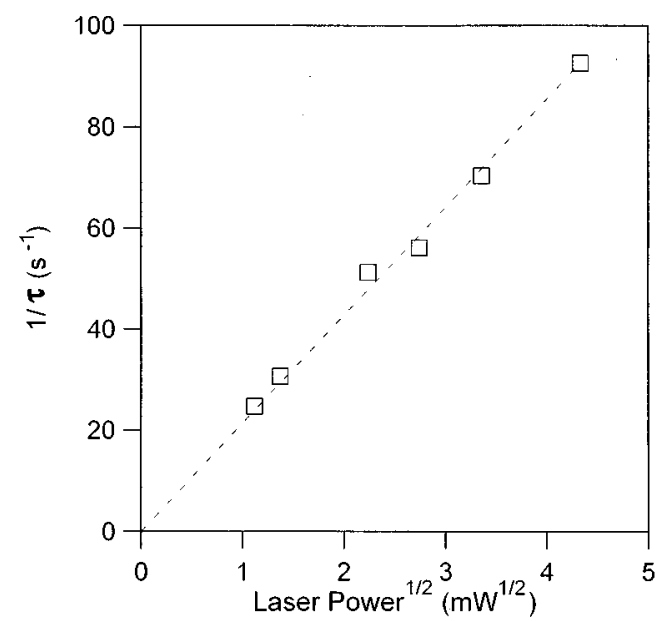

FIG. 4. Dependence of the deexcitation time constant at $t=0$ on the laser power (data taken from Ref. 8). 


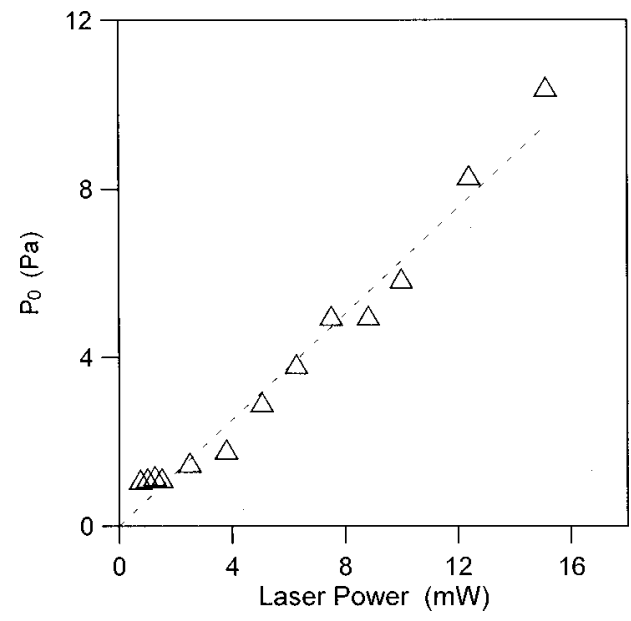

FIG. 5. Dependence of the characteristic pressure $P_{0}$ on the laser power (data taken from Ref. 9).

\section{Pressure quenching of the radiative emission}

The pressure quenching has remained until now the most puzzling fact that has led us finally to think that the multistep-multiphoton mechanism was not the correct explanation for the radiative emission of silicon nanopowder. On the other hand, within the framework of the thermal radiation the effect of the gas is straightforward. The gas offers a new dissipation mechanism for the energy absorbed from the laser. So, the temperature of the particles will be lower and, consequently, the radiative emission will be less intense. The characteristic pressure $P_{0}$ of Eq. (2) is:

$$
P_{0}=\frac{4 \lambda Q_{\mathrm{abs}}}{\alpha h c} \sqrt{\frac{\pi k_{B}}{8}} \frac{R}{c_{v}} \sqrt{m T_{G}} \frac{T_{p}}{T_{p}-T_{G}} I_{L} .
$$

This expression will be tested against the experimental results reported in previous works (Refs. 9 and 10).

First, $P_{0}$ must be proportional to the laser intensity. It is (Fig. 5). Second, it must depend on the gas species through their molecular mass $\left(\mathrm{m}^{1 / 2}\right)$ and their ability to transport energy $\left(R / c_{v}\right)$. Those relationships were found experimentally in Ref. 10. Finally, $P_{0}$ must be proportional to $T_{G}^{1 / 2}$ times $T_{P} / T_{p}-T_{G}$. According to Fig. 6 the experimental results reveal that $P_{0}$ is almost proportional to $T_{G}^{1 / 2}$. This would indicate a very high temperature of the particles. The fitting to the experimental points delivers an unrealistic value of $T_{p} \approx 2600 \mathrm{~K}$, which is far above the fusion temperature of $\mathrm{Si}$. Furthermore, we will show that the particle temperature is not higher than $900 \mathrm{~K}$. The expected evolution of $T_{G}$ at this temperature is far from the experimental behavior (Fig. 6). So, we are led to the puzzling conclusion that the capacity of the gas molecules to dissipate the heat is proportional to the number of collisions and the temperature of the particle, but independent of the energy they have before the collision. Apparently this energy does not contribute to the energetic balance during the collision.

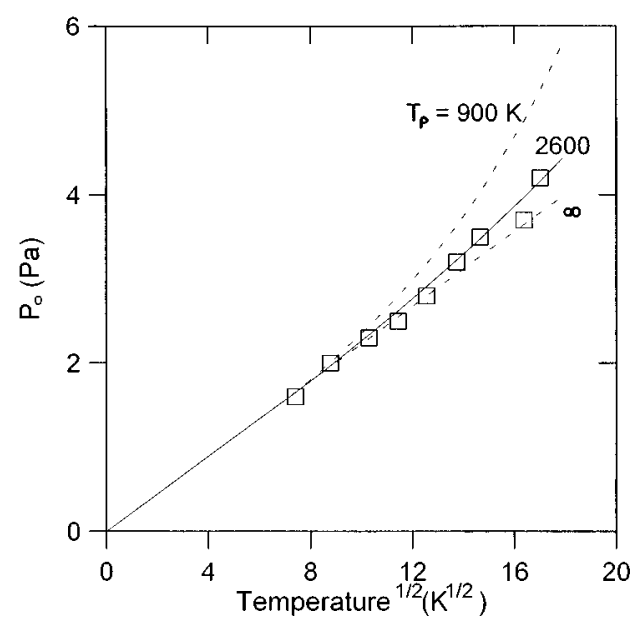

FIG. 6. Dependence of the characteristic pressure on gas temperature. The lines correspond to the evolution expected according to the model for different particle temperatures.

\section{DISCUSSION}

\section{A. Are the particles really independent?}

As pointed out previously, the particles in the powder are not completely independent. In fact, the usual classification of the ceramic raw materials, as the particle size of our powder is well below $1 \mu \mathrm{m}$, should be considered a colloidal system. ${ }^{14}$ In these systems, the inertial forces on the particles are insignificant and the surface forces are dominant. This is really the case in view of the low apparent density of the powder $\left(10 \mathrm{mg} / \mathrm{cm}^{3}\right){ }^{8}$ This value means that the particles occupy only about $1 / 200$ of the powder volume. Consequently, the thermal conductivity of the powder is very small and its contribution to the energy balance is negligible. So, it has not been considered in Eq. (3). Additional proof of this conclusion comes from the dependence of the radiative intensity on the cryostat temperature. From 14 to $300 \mathrm{~K}$, the intensity undergoes only a slight increase by a factor of 2 . So, the particle temperature at the excitation conditions does not follow the variations of the cold finger to which it is glued, indicating negligible thermal conduction.

Another kind of possible mutual influence could arise from the fact that there is a great number of particles inside the volume excited by the laser. One could think that the radiation emitted by the particles could be reabsorbed before it reaches the powder surface. This effect is not included in Eq. (3). In fact, a simple analysis shows that it is negligible. The penetration depth of the laser beam is inversely proportional to the absorption coefficient, $\alpha_{L}$, at the laser wavelength. On the other hand, the emitted radiation will be absorbed after a distance inversely proportional to the absorption coefficient at the emitting wavelength, $\alpha(\lambda)$. Now, as most of the radiation is emitted below the band gap of silicon, $\alpha(\lambda) \ll \alpha_{L}$, the emitted radiation will not be reabsorbed by the emitting particles.

A parameter that is highly sensitive to the particle interactions is the accommodation coefficient [Eq. (5)]. The heat dissipated by the gas collisions can be analyzed more rigorously if we consider the powder as a whole instead of the 
collisions with every particle as we did in Eq. (5). A simple comparison between the absorption coefficient of crystalline $\mathrm{Si}$ at the laser wavelength, $\alpha_{L}=18 \times 10^{3} \mathrm{~cm}^{-1},{ }^{15}$ and the mean radius of the particles (say $r=50 \mathrm{~nm}$ ) indicates that the laser beam passes through many particles before being appreciably absorbed. This means that the gas molecules cannot impinge directly on every one of the excited particles before being thermalized. A gas molecule that enters the excited volume of the powder will collide with many particles before leaving it. This process ensures that the molecule will be thermalized. So, the accommodations coefficient of the powder will be 1 . From this point of view an energy balance of the powder as a whole could be written as:

$$
c_{e} \frac{d T}{d t}=A_{L} I_{L}-\left(A_{R} q_{R}+A_{L} q_{k}\right),
$$

where $c_{e}$ is the heat capacity of the powder, $A_{L}$ is the laser beam section, $A_{R}$ is the total surface area of the emitting particles and $q_{k}$ is the heat gas conduction of Eq. (5) with $\alpha=1$. Following this approach, the functional dependencies on $I_{L}, \lambda, T_{G}$ and $c_{v}$, remain exactly the same as those given in Eqs. (7)-(9).

In the situation of independent particles the materialrelated parameters such as $Q_{\text {abs }}$ or $\epsilon_{i}$ could be estimated within the framework of the Mie theory. ${ }^{16,17}$ However, the apparent density of the powder reveals that the particles have relative mean distances similar to that of the laser wavelength and presumably they are agglomerated similarly as they appear in transmission electron microscopy images. ${ }^{18}$ So, the optical response of each particle is no longer independent from the neighboring particles.

Finally, we will discuss a slight modification of Eq. (7) that takes into account the progressive reduction of the laser intensity as it penetrates the powder:

$$
I_{L}(x)=I_{L} e^{-\alpha_{\mathrm{eff} x}} .
$$

Here $x$ is the distance from the powder surface and $\alpha_{\text {eff }}$ is the extinction coefficient of the powder now treated as a continuum. Every particle is irradiated by the laser at an intensity that depends on its position $x$. So, the observed emission will be:

$$
I_{e}=\int_{0}^{\infty} I_{e}(x) e^{-\alpha_{\mathrm{eff}} x} d x,
$$

where $I_{e}(x)$ is related to $I_{L}(x)$ according to Eq. (7). Now, a first order calculation on $x$ can be made due to the fact that the radiative emission diminishes very quickly with the laser power (Fig. 2). We thus obtain:

$$
\begin{aligned}
I_{e}= & \epsilon(\lambda) \frac{8 \pi h c}{\lambda^{5}} \frac{\lambda k_{B}}{\alpha_{\mathrm{eff}} h c} \frac{4 \sigma \epsilon_{i}}{Q_{\mathrm{abs}}} I_{L}^{1 / 4} \\
& \times \exp \left[-\frac{h c}{\lambda k_{B}}\left(\frac{4 \sigma \epsilon_{i}}{I_{L} Q_{\mathrm{abs}}}\right)^{1 / 4}\right],
\end{aligned}
$$

which shows that the logarithm of $I_{e} / I_{L}^{1 / 4}$ and not $I_{e}$ [as stated in Eq. (7)] should be proportional to $I_{L}^{-1 / 4}$. The experimental results are replotted in Fig. 2 according to this equation.

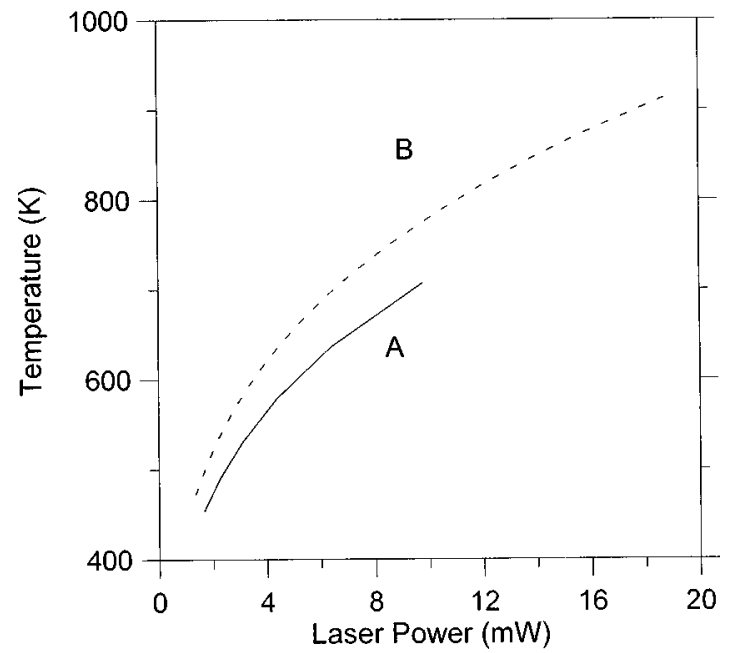

FIG. 7. Temperature of the particles during laser excitation. It has been calculated from the results of Fig. 2 (curve $A$ ) and from those of Ref. 8 (curve $B$ ).

Presumably, the variation of the laser intensity as it penetrates the powder will also modify only slightly Eqs. (8) and (9) in such a way that, within the accuracy of the experimental results, the previous simple relationships with $I_{L}$ still hold.

In conclusion, although, in several instances the particles cannot be considered independent, the general relationships between the experimental variables remain almost unchanged. The main limitation imposed by the mutual influence of the particles is the difficulty in gaining insight into the material parameters.

\section{B. The temperature of the particles}

The temperature of the particles near the powder surface at steady-state conditions can easily be derived from Eqs. (3) and (4).

$$
T_{p}=\left(\frac{Q_{\mathrm{abs}}}{4 \sigma \epsilon_{i}}\right)^{1 / 4} I_{L}^{1 / 4}
$$

If the optical parameters were known, then the temperature could be calculated. However, as stated before, this is not possible with sufficient accuracy. Anyway, if we rewrite Eq. (13) in the form:

$$
\operatorname{Ln} \frac{I_{e}}{I_{L}^{1 / 4}}=A-\frac{h c}{\lambda k_{B}}\left(\frac{4 \sigma \epsilon_{i}}{Q_{\mathrm{abs}}}\right)^{1 / 4} I_{L}^{-1 / 4}
$$

we see that from the slope of the corresponding curve the temperature can be calculated. The result is plotted in Fig. 7. Curve $A$ is the temperature corresponding to the measurements of Fig. 2, whereas curve $B$ corresponds to those results reported in Ref. 8. In view of the excellent linearity of the points in Fig. 2, the values of temperature can be taken with great confidence.

An independent experimental measurement of the particle temperature and the cooling effect due to the gas is provided by Raman measurements. The sample is located in a vacuum chamber and it is illuminated by the laser beam. It 


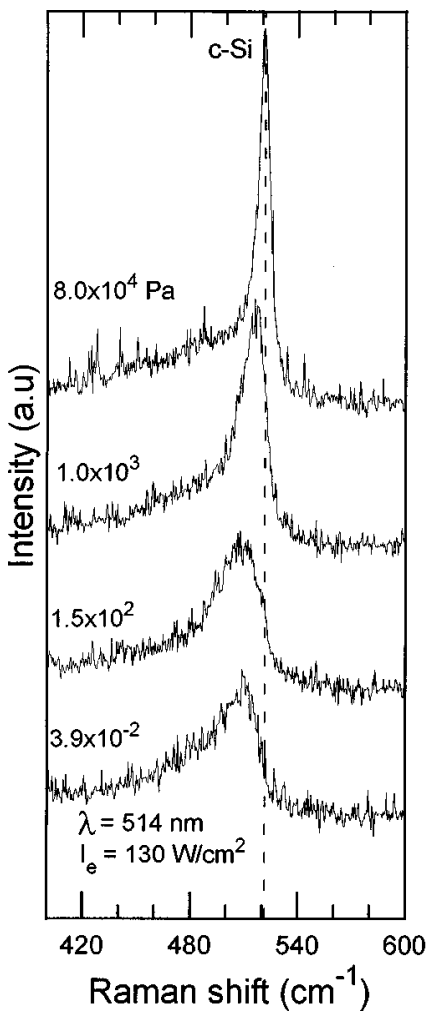

FIG. 8. Raman spectra measured a different gas pressures. The shift of the TO peak is interpreted as due to the heating of the powder.

is known that the transverse optical (TO) peak of $c$-Si shifts from $520.5 \mathrm{~cm}^{-1}$ at $20{ }^{\circ} \mathrm{C}$ to lower wave numbers as the temperature increases according to a linear relationship. ${ }^{19}$

$\omega_{\mathrm{TO}}(T)=\omega_{\mathrm{TO}}(273 \mathrm{~K})-\left(2.81 \times 10^{-2} \mathrm{~cm}^{-1} / \mathrm{K}\right)(T-273)$.

Figure 8 shows the TO peak of silicon powder at different gas pressures. The calculated temperatures of the Si particles from the TO peak shift are presented in Fig. 9. The values are higher than those reached during luminescence experiments due to the higher laser intensity obtained in the Raman microscope.

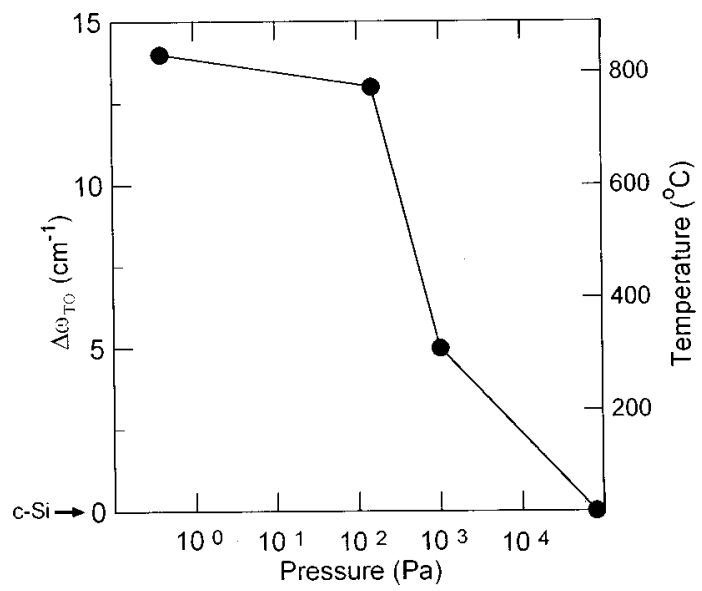

FIG. 9. Temperature of the particles at the excitation conditions of the Raman measurements.

\section{A priori calculation of the temperature}

We now discuss the conditions that make possible the radiative thermal emission in $\mathrm{Si}$ nanoparticles. This will allow us to predict whether such behavior can be expected for other kinds of nanoparticles. The only particle parameters that determine the temperature are the fraction of the incident laser beam intensity that is absorbed by the particle, $Q_{\text {abs }}$, and the integrated emissivity, $\epsilon_{i}$. These parameters can be related to the absorption coefficients at the laser wavelength, $\alpha_{L}$, and in the infrared, $\alpha_{i}$. If we neglect the dispersion of light, then we can state approximately that

$$
Q_{\mathrm{abs}} \approx \alpha_{L} r, \quad \epsilon_{i} \approx \alpha_{i} r .
$$

So, in view of Eq. (14) the temperature is proportional to $\left(\alpha_{L} / \alpha_{i}\right)^{1 / 4}$. Now, in the case of silicon, we can calculate $a$ priori the temperature expected in typical excitation conditions (say, $20 \mathrm{~mW} / 4 \mathrm{~mm}^{2}$ ). If we take $\alpha_{L}=18 \times 10^{3} \mathrm{~cm}^{-1}$ (Ref. 15) and for $\alpha_{i}$ the value of a highly doped silicon at elevated temperatures, $\alpha_{i} \approx 100 \mathrm{~cm}^{-1},{ }^{20}$ we obtain $T_{p}$ $\approx 1400 \mathrm{~K}$. Although this value is far from the experiment it suggests that the radiative thermal emission could have been predicted a priori. A high value of $\alpha_{L} / \alpha_{i} \approx 10^{2}$ is obtained in silicon because the excitation is at a photon energy higher than the band-gap energy.

Now, we can understand why the nanopowder emits the thermal radiation only after it has been dehydrogenated. ${ }^{12}$ This is because with hydrogen the predominant bond is $\mathrm{Si}-\mathrm{H}$, which has an energy in the ultraviolet region, $h \nu_{L}$ $\approx 3.5 \mathrm{eV}$. So, $\alpha_{L}$ is very low before the hydrogen has evolved from the powder.

A simple general criterion for the thermal emission could be the comparison between the band-gap energy $E_{g}$ and that of the exciting photons $\left(h \nu_{L}\right)$. Within our experimental conditions we have not observed any radiation from $\mathrm{BN}$ or $\mathrm{Si}_{3} \mathrm{~N}_{4}$ because, in these cases, $E_{g}>h \nu_{L}$. On the other hand, we believe that it could be possible to detect it in $\mathrm{SiC}$ once dehydrogenated $\left(E_{g}=2.35 \mathrm{eV}\right.$ for the cubic polytype).

Although Eq. (17) suggests that the particle heating is independent of its dimensions, this is not the case. The smaller the particles the more intense the heating for three reasons: (a) when $\alpha_{L}>\alpha_{i}$, as the radius increases $Q_{\text {abs }}$ reaches its limiting value $(\sim 1)$ before $\epsilon_{i}$; (b) the dispersion of the radiation makes $Q_{\text {abs }}$ and $\epsilon_{i}$ smaller than in Eq. (17), this effect being more pronounced at higher wavelengths (that is for $\epsilon_{i}$ ); and (c) at radii greater than about $1 \mu \mathrm{m}$, the particles tend to agglomerate due to their own weight ${ }^{14}$ making the thermal radiation negligible compared with the thermal conduction.

\section{Photoluminescence of porous silicon and related materials}

In the last subsection we analyzed the particle characteristics that make possible the thermal radiation in our experimental conditions for the photoluminescence measurements (under vacuum and at moderate laser intensities): that is, the conditions at which the thermal radiation resembles photoluminescence. For this, a low thermal conductivity is essential. If the measurements are not performed under vacuum the 
necessary laser intensity will increase considerably. As an example, a method to determine the size of soot particles by heating them with a laser pulse has been reported. ${ }^{17}$

Now, the structure of porous silicon and similar materials with reduced thermal conductivity can be locally heated with laser excitation. We believe that some studies reported in literature deal in fact with thermal emission rather than luminescence. Although we have not done extensive research, we have found three papers in which this hypothesis should be taken into account. The first ${ }^{21}$ reports a supralinear dependence of the radiation emitted by laser ablated porous silicon; the second ${ }^{22}$ finds a pressure quenching of the radiative emission of mechanically milled $\mathrm{Si}$ and $\mathrm{SiO}_{2}$ powders, and the third ${ }^{23}$ analyzes the luminescence of porous silicon after a pulsed laser excitation. In this case, the spectra have a two-peak structure. The peak of higher photon energy disappears faster after the laser pulse than the peak in the infrared. This could be consistent with the thermal origin of both. Of course, the information contained in these papers is not sufficient to confirm our suggestion and complementary experiments are needed.

\section{SUMMARY}

An alternative explanation has been proposed to interpret the peculiar 'photoluminescence' observed in PECVD silicon nanopowder. Agreement with the experiment leads to the conclusion that the radiative emission has its origin in the heating of the silicon particles. Although the laser intensities were the usual in photoluminescence experiments, in this case the heating is very important due to the reduced thermal conductivity of the powder as well as the nanometric size of the particles.

Within this new framework, a model involving a set of independent spherical particles has been developed with the aim of predicting the emission characteristics (spectral shape, dynamics, pressure dependence, and nonlinear response) and their dependence on the material parameters (powder size and optical constants). The analysis allows us to determine the particle temperature at the excitation conditions. However it is not possible, at present, to extract the material parameters from the radiative emission, mainly due to the fact that the particles inside the powder are not independent enough.

Although, at present, the only results analyzed in detail are those of our PECVD silicon nanopowder, we believe that the reduced thermal conductivity in similar powders or in porous-silicon related materials can lead to an important heating of the samples in photoluminescence experiments, which can led to erroneous interpretations.

\section{ACKNOWLEDGMENT}

This work has been supported by the Spanish Programa Nacional de Materiales under Contract No. MAT96-1194$\mathrm{C} 02$.

${ }^{1}$ Particle Control for Semiconductor Manufacturing, edited by R. P. Donovan (Decker, New York, 1990).

${ }^{2}$ G. S. Selwyn, J. Singh, and R. S. Bennet, J. Vac. Sci. Technol. A 7, 2758 (1989).

${ }^{3}$ J. Costa, G. Sardin, J. Campmany, and E. Bertran, Vacuum 45, 1115 (1994).

${ }^{4}$ J. Costa, P. Roura, G. Sardin, J. R. Morante, and E. Bertran, Appl. Phys. Lett. 64, 463 (1994).

${ }^{5}$ C. Courteil, J.-L. Dorier, J. Dutta, Ch. Hollentein, and A. A. Howling, J. Appl. Phys. 78, 61 (1995).

${ }^{6}$ L. T. Canham, Appl. Phys. Lett. 57, 1046 (1990).

${ }^{7}$ M. S. Brandt, H. D. Fuchs, M. Stutzmann, J. Weber, and M. Cardona, Solid State Commun. 81, 307 (1992).

${ }^{8}$ P. Roura, J. Costa, G. Sardin, J. R. Morante, and E. Bertran, Phys. Rev. B 50, 18124 (1994)

${ }^{9}$ J. Costa, P. Roura, N. A. Sulimov, G. Sardin, J. Campmany, J. R. Morante, and E. Bertran, Mater. Sci. Technol. 11, 707 (1995).

${ }^{10}$ P. Roura, J. Costa, J. R. Morante, and E. Bertran, J. Appl. Phys. 81, 3290 (1997).

${ }^{11}$ H. Hofmeister, J. Dutta, and H. Hofmann, Phys. Rev. B 54, 2856 (1996).

${ }^{12}$ J. Costa, P. Roura, A. Canillas, E. Pasqual, J. R. Morante, and E. Bertran, Thin Solid Films 276, 96 (1996).

${ }^{13}$ J. E. Daugherty and D. B. Graves, J. Vac. Sci. Technol. A 11, 1126 (1993).

${ }^{14}$ J. S. Reed, in Principles of Ceramic Processing, 2nd. ed. (Interscience, New York, 1994), p. 70.

${ }^{15}$ Data in Science and Technology, Semiconductors, Group IV Elements and III-V Compounds, edited by O. Madelung (Springer, Berlin, 1991).

${ }^{16} \mathrm{M}$. Kerker, The Scattering of Light and Other Electromagnetic Radiation, (Academic, New York, 1969).

${ }^{17}$ P. Roth and A. V. Filippov, J. Aerosol Sci. 27, 95 (1996).

${ }^{18}$ J. Dutta, W. Bacsa, and Ch. Hollenstein, J. Appl. Phys. 77, 3729 (1995).

${ }^{19}$ T. R. Hart, R. L. Aggarwal, and B. Lax, Phys. Rev. B 1, 638 (1970).

${ }^{20}$ P. J. Timans, J. Appl. Phys. 74, 6353 (1993).

${ }^{21}$ D. P. Savin, Ya. O. Roizin, and D. A. Demchenko, Appl. Phys. Lett. 69, 3048 (1996)

${ }^{22}$ T. D. Shen, I. Shmagin, C. C. Coch, R. M. Kolbas, Y. Fahmy, C. Bergman, R. J. Nemanich, M. T. McClure, Z. Sitar, and M. X. Quan, Phys. Rev. B 55, 7615 (1997).

${ }^{23}$ S. Puccini, V. Pellegrini, M. Labardi, F. Fuso, M. Allegrini, A. Diligenti, A. Nannini, and G. Pennelli, Nuovo Cimento D 18, 1149 (1996). 